\title{
Hydrodynamic Complexity Induced by the Pulsing Flow Field in USP Dissolution Apparatus 4
}

\author{
Deirdre M. D'Arcy ${ }^{1, ~}{ }^{\text {, }}$ Bo Liu' ${ }^{1}$,Tim Persoons ${ }^{2}$, and Owen I. Corrigan ${ }^{1}$ \\ 'School of Pharmacy and Pharmaceutical Sciences, Trinity College Dublin, Dublin 2, Ireland \\ ${ }^{2}$ Department of Mechanical and Manufacturing Engineering, Trinity College Dublin, \\ Dublin 2, Ireland
}

\begin{abstract}
USP dissolution Apparatus 4 can have a pulsing or non-pulsing flow, with most commercial apparatus employing a pulsing flow. Overall, a low velocity flow field is present, particularly in the larger 22.6-mm diameter cell. Dissolution data, computational fluid dynamics (CFD), and imaging methods are used and discussed to investigate the effects of low velocity pulsing flow on dissolution. Local velocity patterns, density gradients, and the effect of flow rate on particulate dispersion can all contribute to the observed dissolution rate. In silico simulation tools and imaging techniques have proved useful in determining local hydrodynamics and, in some cases, their effect on observed dissolution rates. Methods to investigate concentration gradients at the dissolving surface are required to investigate further the effect of hydrodynamics on the dissolution process. In conclusion, it is necessary to take local flow patterns and velocities into account, rather than overall flow rate alone, when interpreting dissolution rate data in the flow-through apparatus.
\end{abstract}

\section{INTRODUCTION}

$\mathrm{H}$ ydrodynamics in USP dissolution Apparatus 4 have been analyzed theoretically using computational

fluid dynamics (CFD) and imaging techniques such as magnetic resonance imaging (MRI) (1-4). An important point to recognize when considering hydrodynamics in the flow-through apparatus is the low-velocity operating conditions. Although low-velocity regions are present at the center of the vessel base in both paddle and basket apparatus, in general, fluid velocities in the flow-through cell are lower than those present in the paddle and basket apparatus at typical operating conditions. For example, previous CFD simulations have predicted that the maximum velocity at $1 \mathrm{~mm}$ from a compact positioned at the center of the base of the paddle apparatus at $50 \mathrm{rpm}$ ranged from almost zero to maximum values of $4.9 \times 10^{-2}$ to $6.7 \times 10^{-2} \mathrm{~ms}^{-1}$, with the velocity increasing radially from this location. Maximum simulated velocities relative to the location of a rotating compact in the basket of the basket apparatus at $50 \mathrm{rpm}$ were approximately $2.6 \times 10^{-2} \mathrm{~ms}^{-1}(5)$. On the other hand, in the smaller $12-\mathrm{mm}$ cell of USP 4 flow-through apparatus at $17 \mathrm{~mL} / \mathrm{min}$, the predicted maximum velocity was approximately $1.4 \times 10^{-2} \mathrm{~ms}^{-1}$ in a simulation of a cell containing a vertical compact (3). In contrast to simulated velocity data from the paddle and basket apparatus, this maximum value only occurs during the peak flow velocity generated by the pulsing flow field. As pharmacopeial recommended flow rates range from 4 to $16 \mathrm{~mL} / \mathrm{min}(6,7)$ and velocities are faster in the $12-\mathrm{mm}$ cell than in the $22.6-\mathrm{mm}$ cell due to the reduced cross-sectional area, this value of $1.4 \times 10^{-2} \mathrm{~ms}^{-1}$

\footnotetext{
*Corresponding author
}

can be considered a representative maximum velocity present in the flow-through apparatus at typical operating conditions. The size, shape, and orientation of a dosage form in the cell will naturally also affect the cross-sectional area available for fluid to traverse the cell, with a horizontally orientated compact likely to generate a slightly higher velocity than the model with a vertically orientated compact. In the $22.6-\mathrm{mm}$ diameter cell operated at $8 \mathrm{~mL} / \mathrm{min}$, the average fluid velocity through the cell is $0.033 \times 10^{-2} \mathrm{~ms}^{-1}$ in a cell containing no dosage form. For a pulsing flow, in a cell containing a compact, the maximum simulated fluid velocity at $1 \mathrm{~mm}$ from the compact surface was $0.13 \times 10^{-2} \mathrm{~ms}^{-1}(3)$.

In addition to the typical flow rates outlined in the pharmacopeias, it is also stated that flow may be constant or pulsing. A pulsing flow must consist of $120( \pm 10)$ pulses per minute and follow a sinusoidal flow profile $(6,7)$. The flow profile from a typical piston pump is detailed in Figure 1, with the outflow following a half-sine-wave profile during the pump discharge phase (0-0.25 s) and zero outflow during the pump suction phase (during suction from the reservoir into the pump). Figure 1 also depicts vectors of velocity magnitude from a simulation of flow around the curved side of a compact in the 12- $\mathrm{mm}$ diameter cell at $17 \mathrm{~mL} / \mathrm{min}$, illustrating the simulated velocities present near the maximum inflow velocity in a pulsing flow and during the zero inflow velocity period. The situation of flow past the planar surface of the compact simulated under the same flow conditions was previously presented (3).

The variation in fluid velocity distribution in the cell of USP Apparatus 4 arise from both (1) variation of input flow rates and (2) variation of velocity in the cell over each pulse at a specific flow rate. The magnitude and nature of 


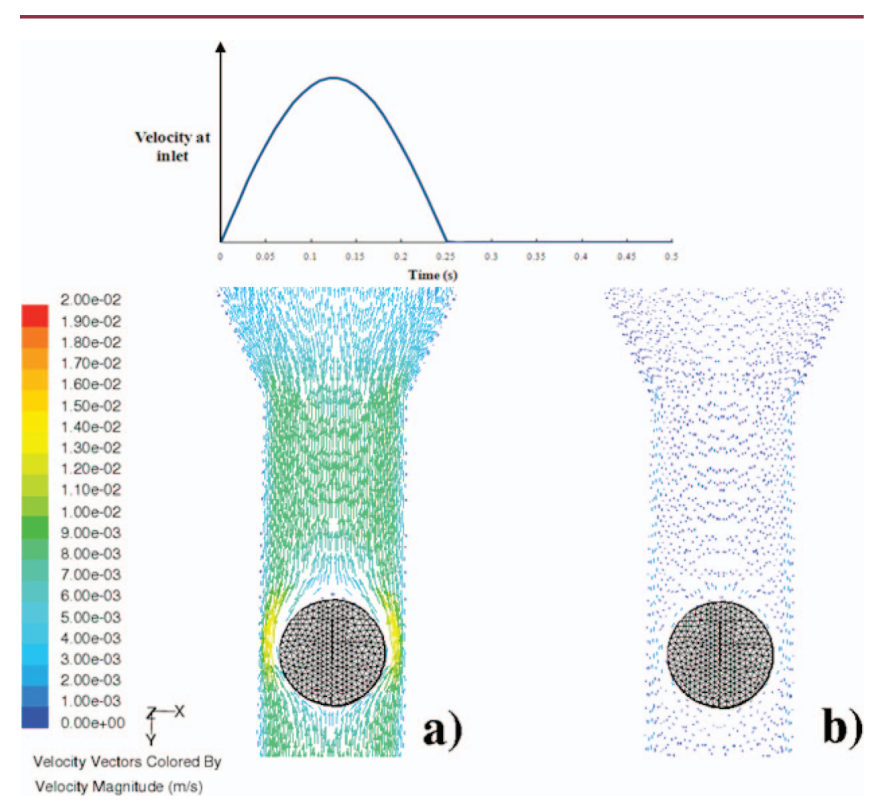

Figure 1. Profile of fluid velocity vs. time used to simulate the cell inflow from the piston pump in USP dissolution Apparatus 4. Also shown are vectors of velocity magnitude $\left(\mathrm{ms}^{-1}\right)$ from a simulation of flow around the curved side of a compact in the 12- $\mathrm{mm}$ diameter cell at $17 \mathrm{~mL} / \mathrm{min}$ at (a) $0.15 \mathrm{~s}$ (near peak inflow) and (b) $0.35 \mathrm{~s}$ (during zero inflow period).

the variation in fluid dynamics further depend on the cell size used. As dissolution rate is intrinsically linked to local hydrodynamics through the effect of hydrodynamics on the concentration gradient, an insight into the hydrodynamics in the flow-through cell is crucial for interpretation of dissolution rate data. The current work considers the effects of features present in a low-velocity pulsing flow field on dissolution rates in USP dissolution Apparatus 4. As the pulsing flow includes periods of zero inflow, a free convection system was also simulated.

\section{METHODS}

Hydrodynamic and Mass Transfer Simulations

CFD was used to generate 3D simulations of hydrodynamics in the 22.6-mm diameter cell at 8, 16, and $43 \mathrm{~mL} / \mathrm{min}$, using the method previously described (3). The model contained a cylinder within the cell, representing a dissolving compact, at the location of the tablet holder, with the flat planar surfaces orientated vertically. One pulse through the cell was simulated and consisted of both the pump discharge and pump suction phases.

The 2D simulation of velocity and mass transfer in the free convection system was generated using the speciestransfer model.

The free convection system consisted of a jar filled with dissolution medium, with a cylindrical compact suspended from the lid of the jar in a vertical orientation. As only one planar surface was exposed to the dissolution medium, only one planar surface was included in the species transfer simulation. Species transfer does not simulate the dissolution process, but it can be used to simulate a situation where a saturated solution of solute is present at the dissolving surface, thereby simulating the effect of the dissolution process on local hydrodynamics. The CFD software package FLUENT (ANSYS, Inc., NH, USA) was used to generate the fluid dynamics and species-transfer simulations.

\section{Dissolution Studies}

Dissolution tests were carried out in the flow-through apparatus 22.6-mm diameter cell at 8,16 , and $43 \mathrm{~mL} / \mathrm{min}$, using the method previously described (3). In summary, compacts consisted of $500 \mathrm{mg}$ of benzoic acid and were $13 \mathrm{~mm}$ in diameter and approximately $3 \mathrm{~mm}$ in height. The compacts were coated with paraffin wax, leaving only one of the flat, planar surfaces exposed to the dissolution medium. The compacts were placed in the tablet holder of the flow-through cell with the exposed planar surface orientated vertically. The dissolution medium was $0.1 \mathrm{M}$ $\mathrm{HCl}$, and dissolution tests were carried out at $37^{\circ} \mathrm{C}$ using a closed system configuration, with a reservoir volume of $900 \mathrm{~mL}$.

\section{Shadowgraph Imaging}

The shadowgraph imaging method used was previously described (8). In brief, it involves using a double-frame camera with a high-magnification lens to capture images of a particle-containing fluid against a background of uniform pulsed illumination, thereby being a noninvasive technique. The field of view had a resolution of $6 \mu \mathrm{m}$ per pixel, (magnification 3.33:1). The field of view was limited to a $6 \times 6 \mathrm{~mm}$ section at the lower right-hand side of the flow-through cell. Particle sizes and velocities were determined using LaVision DaVis 7.2.2 software (LaVision $\mathrm{GmbH}$, Göttingen, Germany). A schematic diagram of the sampling window and experimental setup of the powder cell used in the current work is presented in Figure 2a.

\section{RESULTS AND DISCUSSION: \\ Local Hydrodynamics: Flow Reversal, Low-Velocity} Periods, and Local Fluid Dynamics Variations

Simulated images of velocity vectors near the curved surface of the compact at $16 \mathrm{~mL} / \mathrm{min}$ are shown in Figure 3. Figure 3 a shows velocity vectors at $0.06 \mathrm{~s}$, and Figure $3 \mathrm{~b}$ at $0.2 \mathrm{~s}$. The inflow velocities to the cell are similar at these two time points (flow profile, Figure 1); however, it is clear that flow reversal is commencing near the compact surface in Figure $3 \mathrm{~b}$ (inset). An interesting hydrodynamic feature of pulsing flow in USP dissolution Apparatus 4 is that boundary layer separation followed by flow reversal occurs during the decreasing velocity period of the pump discharge phase. Due to viscous friction, a hydrodynamic boundary layer develops during the increasing velocity period, leading to a separation between high kinetic energy fluid in the bulk flow and low energetic fluid near the wall. Because of this kinetic energy difference, the boundary layer is subjected to an adverse pressure gradient when the flow decelerates towards the 


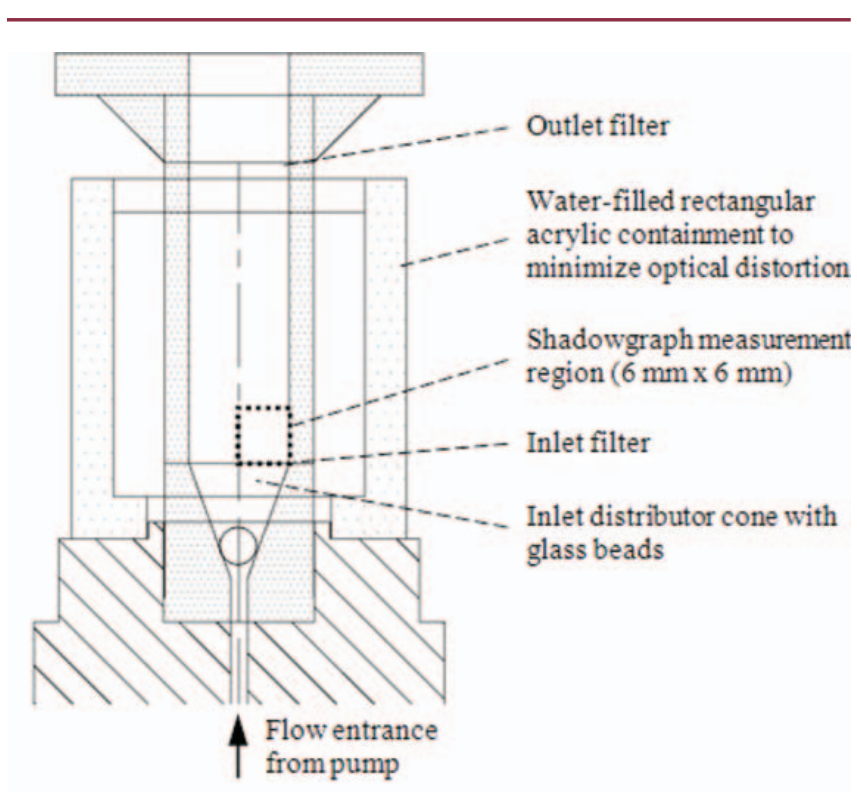

a)

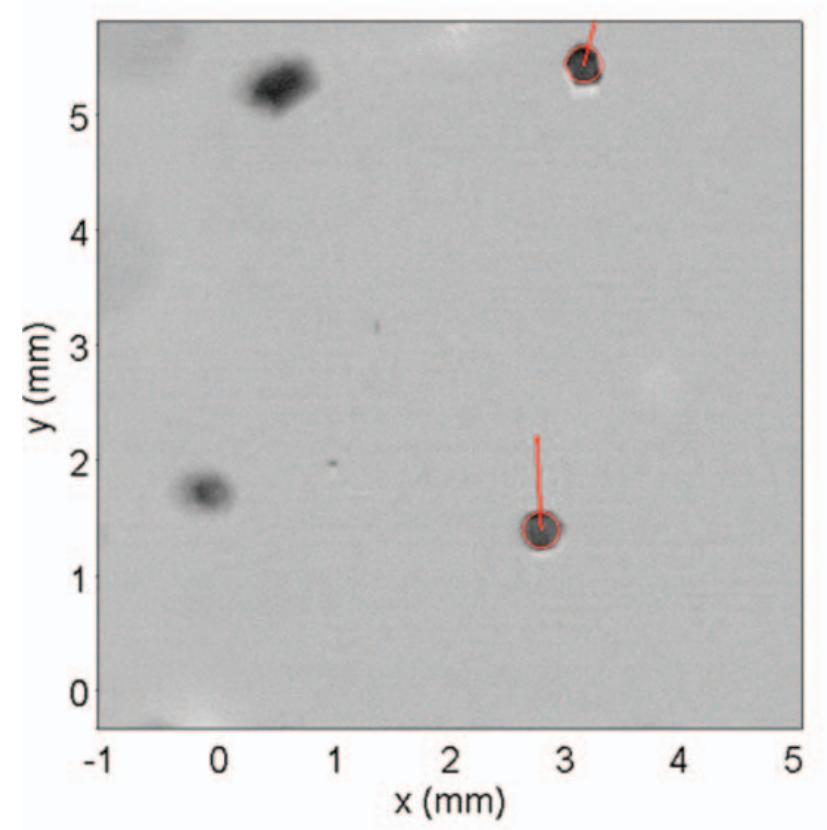

b)

Figure 2. (a) Schematic diagram of the shadowgraph imaging set up for the 12-mm diameter powder cell, showing the sampling window used in the shadowgraph imaging. (b) A typical image generated using the shadowgraph imaging method, where a particle is visualized with the red arrow showing velocity magnitude and direction. In this case, the particle is moving vertically upwards.

end of the pump discharge phase. This pressure gradient in turn causes the flow to detach from the wall, creating a region of instantaneous flow reversal. This feature was previously presented (3) and was observed in a simulation

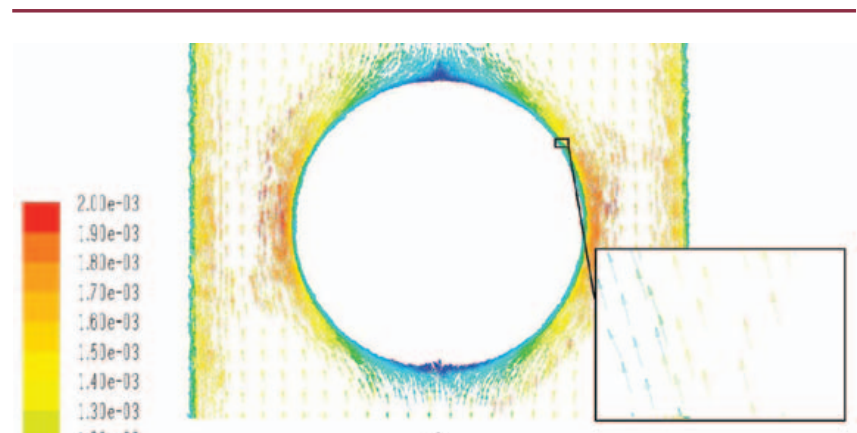

a)

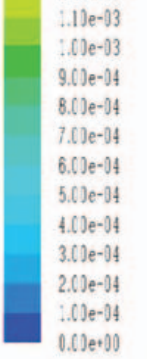

$t^{-x}$

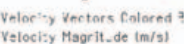

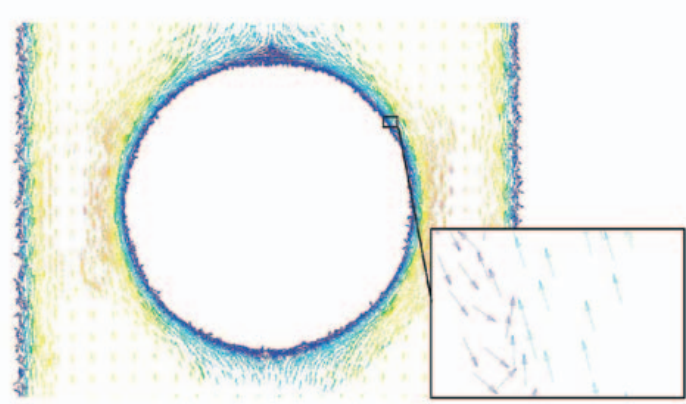

b)
Figure 3. Vectors colored by velocity magnitude $\left(\mathrm{ms}^{-1}\right)$ from a 3D simulation of hydrodynamics near the curved surface of the compact in the 22.6- $\mathrm{mm}$ diameter cell at $16 \mathrm{~mL} / \mathrm{min}$, at (a) $0.06 \mathrm{~s}$ and (b) $0.2 \mathrm{~s}$.

of $16 \mathrm{~mL} / \mathrm{min}$ at $0.1875 \mathrm{~s}(2)$, and at $8 \mathrm{~mL} / \mathrm{min}$. In the current work, flow reversal occurred at all three flow rates simulated. At each flow rate, the boundary layer separation had occurred and flow reversal was commencing by $0.2 \mathrm{~s}$. The discrepancy in the time for boundary layer occurrence can be attributed to different hydrodynamics, as the compact shape was different in both simulations (a curved surface in the work by Kakhi, whereas the current work used a flat planar surface). Furthermore, because the work by Kakhi (2) used a time step of $0.00025 \mathrm{~s}$, compared with $0.01 \mathrm{~s}$ in the current work, the simulation was more refined over this pump discharge phase but did not include simulation of flow over the following pump suction phase. Evidence for residual fluid motion during the pump suction phase, particularly at higher flow rates in the smaller 12-mm diameter cell, was previously demonstrated using both CFD simulations and flow visualization studies (3).

Flow reversal is a well-known phenomenon in oscillating laminar flows (9). For simple geometries such as an infinite cylindrical pipe, the velocity profile subject to an oscillating pressure gradient can be determined analytically, by directly solving the incompressible Navier-Stokes equations. At higher Stokes numbers $\left(S t=2 \pi f R^{2} / v>10\right.$, where $f$ is the pulsation frequency, $R$ is the pipe radius, and $v$ is the kinematic viscosity), the velocity profile becomes flatter rather than parabolic, and displays features of both an overshoot near the wall and flow reversal at the end of the half period. For a pulsed flow 


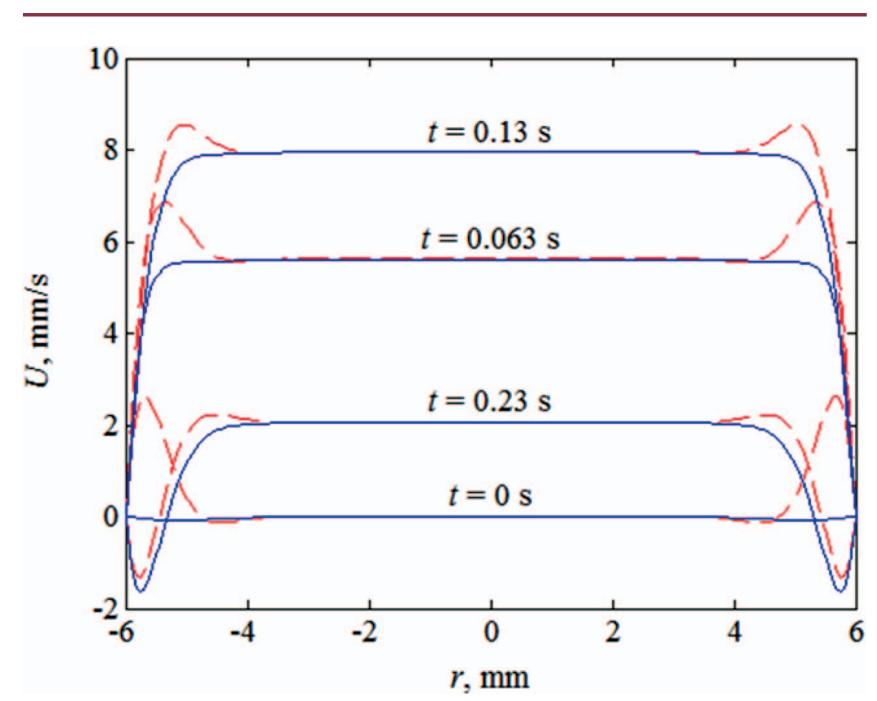

Figure 4. Time evolution of the flow velocity profile in a cylindrical pipe subject to a (red) sine-wave and (blue) semi-sine wave pressure gradient corresponding to the profile in Figure 1 (diameter $2 \mathrm{R}=12 \mathrm{~mm}, \mathrm{f}=2 \mathrm{~Hz}$, $\left.\rho=1000 \mathrm{~kg} / \mathrm{m}^{3}, \mathrm{v}=1 \times 10^{-6} \mathrm{~m}^{2} / \mathrm{s}, \mathrm{St}=450\right)$, numerically obtained by solving the simplified $\mathrm{N}$-S equations for axisymmetric laminar flow.

(semi-sine wave input), rather than an oscillating flow (full sine wave input), the Navier-Stokes equations must be solved numerically. Taking an example of water flowing through a 12-mm diameter pipe, oscillating at a frequency of $2 \mathrm{~Hz}$, the flow profile reveals an overshoot near the wall, in particular at higher velocity periods of the flow, and flow reversal near the wall near the time point of the reversal of the oscillating wave (Figure 4). In the pulse flow situation, the overshoot of a typical oscillating flow disappears, since the input flow comes to a standstill during the pump suction phase. However, flow reversal still occurs near the end of the inflow phase (pump discharge phase) (Figure 4).

The relevance of boundary layer separation, and to an extent residual fluid motion, can be presented in terms of the concentration gradient at the surface. In considering the simplified situation of a uniform constant velocity calculated from average flow rate and cross-sectional area, the concentration gradient would be constant. In the more complex situation of a pulsing flow field, the concentration gradient would vary over the course of the pulse, an effect that might be anticipated to occur in tandem with the flow profile of the pulse. However, with the insight provided by CFD simulations, there is evidence to suggest that hydrodynamics close to the compact consist of local features such as flow reversal and, in some cases, recirculating flows superimposed on the background variation of velocity with the pulsing flow. Thus, the concentration gradient varies over the course of the pulse, and the impact of the changing direction of flow on local concentration gradients is largely unknown.

A flow reversal close to the cell wall was also simulated and was experimentally observed using MRI studies (4).
Table 1. Dissolution Rate $\left(\mathrm{mg} \mathrm{min}^{-1} \mathrm{~cm}^{-2}\right)$ from One Exposed Planar Surface of a Benzoic Acid Compact Orientated Vertically in the Tablet Holder of the 22.6- $\mathrm{mm}$ Diameter Cell, in USP Flow-Through Apparatus 4

\begin{tabular}{|c|c|}
\hline Flow Rate (mL/min) & Average Dissolution Rate $\left(\mathrm{mg} \mathrm{min}^{-1} \mathrm{~cm}^{-2}\right)$ \\
\hline 8 & $0.094 \pm 0.008$ \\
\hline 16 & $0.095 \pm 0.007$ \\
\hline 43 & $0.102 \pm 0.003$ \\
\hline
\end{tabular}

Dissolution medium $0.1 \mathrm{M} \mathrm{HCl}$

Temperature variations at the cell wall were considered a contributing factor to the MRI observations (4); however, the CFD simulations were temperature invariant.

In addition to the effect of flow reversal at all flow rates at the compact surface on the concentration gradient, during each pulse there is a low-velocity period regardless of flow rate. As a result, the hydrodynamics relevant to dissolution (i.e., near the compact surface) may result in the concentration gradient varying less than might be expected with an increase in flow rate. In the current work, there were no significant differences in dissolution rates at 8,16 , and $43 \mathrm{~mL} / \mathrm{min}$, because the standard deviations of dissolution rate at each flow rate were overlapping, although a trend of a slight increase in dissolution rate with flow rate (Table 1 ) is suggested. This lack of a significant effect of increasing flow rate on dissolution rate was previously noted in a system using salicylic acid in $\mathrm{pH} 7.4$ phosphate buffer (10) and was only observed in the larger 22.6-mm diameter cell. In the faster fluid environment of the $12-\mathrm{mm}$ diameter cell, dissolution rates increased with increasing flow rates.

A recent detailed investigation using MRI to study hydrodynamics in a USP Apparatus 4 cell also reported very low velocities present in the cell at some points of the pulse (4). Variation of hydrodynamics with flow rate was presented for several flow rates in both the 12- and 22.6-mm diameter cell, with differences in observed results between both cells discussed. In both cells, a discontinuity in the sinusoidal flow cycle was noted at the point of the pulse where the piston pump changed from fluid acceleration to relaxation, with evidence presented for notable residual flow during the pump suction phase. Furthermore, a negative flow was observed in certain regions of the cell. Tablet orientation and location within the cell impacted significantly on the local fluid velocities to which a tablet was exposed, with both axial and radial velocities investigated.

These observed local hydrodynamic characteristics such as radial velocities, velocity variability, and residual flow therefore differ according to cell size and tablet location and orientation. In the current work, the effect of local hydrodynamics, flow reversal, and overall changing velocities over the course of the pulse were of greater significance than the overall flow rate. This was manifested 
in the observation that there was no increase in dissolution rate with flow rate, suggesting that the sum of local hydrodynamic forces had an equivalent overall effect on the effective concentration gradient at each flow rate. This supports the concept that the local hydrodynamic characteristics at a nondisintegrating tablet surface need to be considered rather than the overall flow rate, in terms of associating hydrodynamics with the concentration gradient driving the dissolution process. It is speculated that the increase in shear rate in the first half of the pulse is not adequate to affect the concentration gradient significantly following the effects that downward flow reversal and subsequent low-velocity period have in the preceding and subsequent periods of each pulse under these low flow rates. It is not yet clear what increase in velocity is required to result in an increase in dissolution rate, as the local hydrodynamic environment is also subject to effects from solute solubility and density.

\section{Density Gradients}

A second important feature to consider in dissolution testing in a low-velocity pulsing flow is the effect of the density of the saturated solution. This parameter is influenced by both the density and solubility of the solute. In an environment where there is no forced convection, free convection can arise. Free convection can be induced by both temperature induced density gradients and density gradients due to the difference in density between a solution and the solvent. If the flow-through cell is considered to be temperature invariant (e.g., in a standard jacketed cell), free convection can be due primarily to the density gradient induced by the density difference between a saturated solution near the dissolving surface and the solvent. There may also be some buoyancy effect at the dissolving surface generated by the heat of solution. The impact of natural convection in a low-velocity environment was presented previously (11), with the effect increasing with increased solubility of solute.

Consider a dissolving surface in a closed-cell environment with no forced convection or mechanically induced flow. Under the influence of gravity, a more dense solution flows downwards. This affects the solute distribution within the cell, but also affects the velocity at the dissolving surface through generation of free convection. The relevance of this free convection at the dissolving surface depends on the location and orientation of the dissolving surface. For example, for a vertically orientated dissolving surface suspended in the dissolution medium, free convection generated by density gradients can flow downwards over the dissolving surface. The velocity generated by the free convection can affect the concentration gradient at the surface, but the pattern of solute distribution in the bulk system does not have a significant immediate effect on the concentration gradient at the surface. This is because the more dense solution flows downwards, away from the dissolving surface, and therefore the bulk concentration in the region

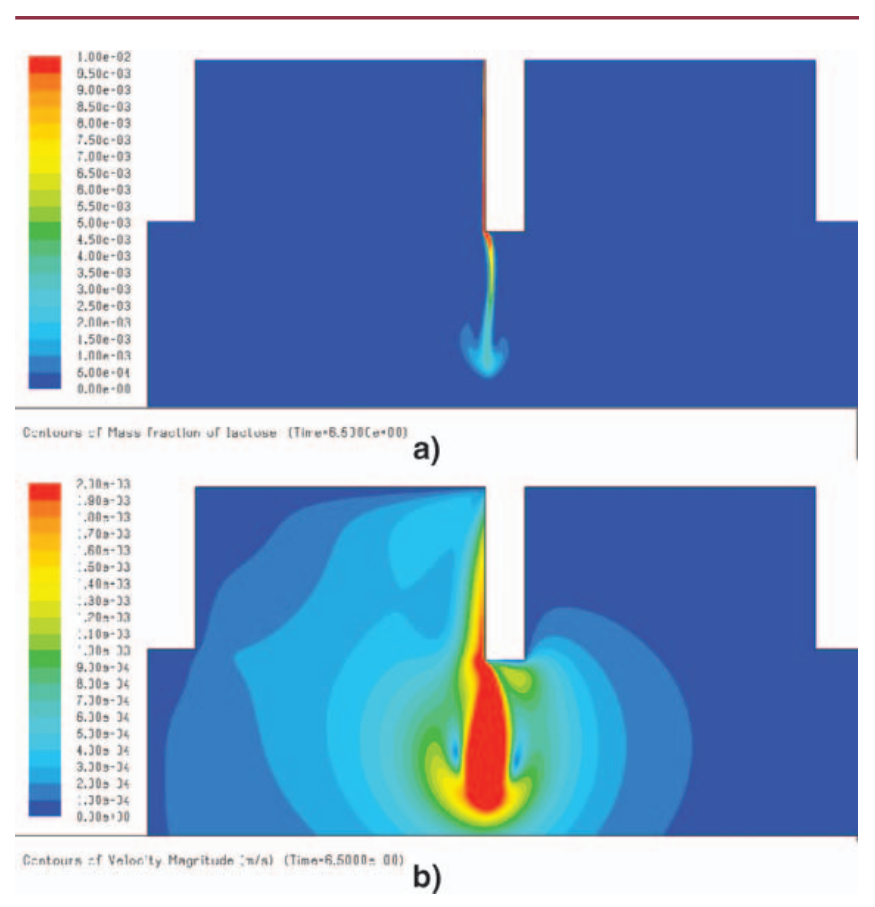

Figure 5. (a) Simulated concentration gradient in the free convection system with a boundary condition at the vertical planar surface of a constant saturated solution of lactose monohydrate in the $0.1 \mathrm{M} \mathrm{HCl}$ dissolution medium, and (b) the velocity magnitude contours $\left(\mathrm{ms}^{-1}\right)$ generated by the effect of gravity on the density gradient from the saturated solution of lactose monohydrate in $0.1 \mathrm{M} \mathrm{HCl}$ at the vertical planar surface.

of the dissolving surface is only affected by diffusion from the solution near the surface and from the base of the cell where the more concentrated solution collects. Therefore, the free convection generated increases the concentration gradient at the surface and the overall effect is an increased dissolution rate. In contrast, if a dissolving surface is located at the base of a free convection cell, it is more difficult for the density gradient to generate a significant free convection velocity, as the more concentrated solution pools at the base of the cell where the dissolving surface is located. This reduces the local density gradient and hence the free convection generated. Furthermore, the more dense pooled solution decreases the concentration gradient at the dissolving surface. Therefore, the effect of the density gradient would be to reduce dissolution overall for a dissolving surface located at the base of a cell, and to increase dissolution for a dissolving surface suspended within a cell. Figure 5 illustrates a simulation in a closed, free convection system (a jar) with a vertical dissolving surface suspended from the lid of the jar. The current work is a simulation of hydrodynamics and species transfer in the jar with a defined constant source of lactose monohydrate saturated solution at the vertical dissolving surface of the lactose monohydrate compact. The simulation does not include any forced convection and therefore the predicted hydrodynamics arise from free convection alone. Figure $5 a$ depicts the simulated concentration gradients in the jar 
of lactose monohydrate from the dissolving vertical planar disc surface in $0.1 \mathrm{M} \mathrm{HCl}$, and Figure $5 \mathrm{~b}$ depicts the velocity magnitude contours generated by the effect of gravity on the density gradient. The downward natural convection generated from the more dense saturated solution present at the vertical dissolving surface is evident in this $2 \mathrm{D}$ simulation.

In a low-velocity environment, free convection interacts with forced convection. In the flow-through apparatus, as there is a low-velocity environment overall with periods of zero-velocity input, there is potential for a significant effect from natural convection. Furthermore, as the flow direction is upward, this is directly countering the downward free convection.

The effect of density gradients on dissolution in a lowvelocity flow-through environment, including orientation relative to gravity and flow direction, was previously simulated and described (11). Furthermore, an increased dissolution rate of slightly soluble benzoic acid in $0.1 \mathrm{M}$ $\mathrm{HCl}$ in a free convection system compared with that at $8 \mathrm{~mL} / \mathrm{min}, 22.6-\mathrm{mm}$ diameter cell in the flow-through apparatus was reported (3). Simulated results in that situation suggest that intermittent downward flow due to flow reversal rather than natural convection reduced flow at the surface in the flow-through apparatus. A recent study (12) presented a detailed analysis of the interaction between forced and natural convection in the flow-through apparatus, suggesting that solubility of the solute plays a major role in the generation of natural convection at the dissolving surface.

The hydrodynamic environment at a dissolving surface suspended in the flow-through cell, therefore, is influenced by the overall pulsing flow, local effects such as flow reversal, and downward free convection, when the solution density is high enough to generate free convection against the forced convection environment. As the fluid velocity at a surface affects the hydrodynamic boundary layer thickness, and the hydrodynamic boundary layer thickness affects the diffusion boundary layer thickness, it is evident that changing local fluid velocities affects the dissolution rate. As illustrated in the theory outlined above, the nature of these changing local fluid velocities does not have a simple relationship with the overall pump flow rate.

\section{Dissolution of Particulate Systems}

To this point, we have been considering a static dissolving surface. In many cases dissolution testing in USP Apparatus 4 involves particulate systems, from pure API to disintegrated dosage forms. A comprehensive analysis on the effect of particle size and density on dissolution in the flow-through cell was presented (8). The velocity relevant to particulate dissolution is the relative velocity; in other words, as the fluid moves, a particle is free to move with the fluid. However, the particle velocity is rarely identical to the fluid velocity, depending on particle size, shape and density as, for a spherical particle, the velocity is equal to the sum of a number of forces including drag force, buoyancy forces, and fluid acceleration (8).

For a spherical particle, the relative velocity can be expressed in terms of the particle Reynolds number, $R e_{p}$ :

$$
R e_{p}=\rho_{f}\left|U_{f}-U_{p}\right| \frac{d_{p}}{\mu_{f}}
$$

where $\rho_{f}$ is the fluid density, $U_{f}$ and $U_{p}$ are the fluid and particle velocities, respectively, $d_{p}$ is the diameter of the particle, and $\mu_{f}$ is the fluid viscosity.

Dissolution is then related to the $R e_{p}$ as follows:

$$
\frac{k d_{p}}{D}=2+0.6\left(\frac{\left|U_{f}-U_{p}\right| d_{p}}{v_{f}}\right)^{0.5}\left(\frac{v_{f}}{D}\right)^{0.33}
$$

where $k$ is the mass transfer coefficient, $D$ is the diffusion coefficient of the solute in the solvent, and $v_{f}$ is the kinematic viscosity of the fluid. The change in particle mass with time (dissolution rate) is then related to the solubility of the dissolving substance, the mass transfer coefficient $(k)$, and the surface area of the dissolving particle.

Although this theory is relatively straightforward, most particulate dissolution systems involve a particle size distribution. Furthermore, particle motion is bound by the flow-through cell boundary. In other words, rather than having a constant relative velocity, there are periods of absolute velocity when a particle is held at the upper or lower cell boundary due to buoyancy effects.

Therefore, rather than one initial relative velocity applied to one initial particle size, a range of relative velocities is present. Furthermore, as particles dissolve, the particle size distribution changes over time, and therefore at the same flow rate the relative fluid velocities also change over time. In the pulsing flow of the flow-through apparatus, the relative velocity value represents the difference between fluid and particle velocity over the course of the pulse. As the fluid velocity increases, the particle velocity also increases, and as the fluid velocity reduces to zero, the particle is free to move downward under gravity, if it is denser than the fluid and the buoyancy force overcomes viscous friction. It can be seen, therefore, how particle density plays a role in the relative fluid velocity generated by a particulate system.

An increase in flow rates leads to an increase in absolute velocity, which also leads to an increase in particle velocity. Therefore, in theory, relative velocity is not predicted to vary with flow rate for a given particle size. According to eq 2 , a constant relative velocity suggests a constant dissolution rate; however, an increase in dissolution rate with flow rate for particulate systems was noted (8). A constant relative velocity for a given particle size assumes an idealized situation of an independent particle moving either in one direction with the fluid or in the direction opposite to the fluid flow during zero- or low-velocity 
periods, when the particle is more dense than the fluid, and the flow direction is opposite gravity. On observing particulate dissolution in USP Apparatus 4, however, three additional phenomena are thought to contribute to the hydrodynamic environment of the dissolving particles. Firstly, particles are moving in a three-dimensional space, and therefore there is some radial movement in addition to upward and downward movement. The extent of this radial movement may be affected by flow rate. Secondly, unless the system is very dilute, particles are not always independent spheres and can, for example, form a loose cake at the base (or top) of the cell. In this situation, particle motion is inhibited by adjacent particles, and the local concentration gradient is affected by dissolution of adjacent particles, the result being to decrease the dissolution rate. An increase in flow rate can lead to increased motion and dispersal of this cake, leading to a system gradually approaching the scenario of independently dissolving particles. An increase in flow rate, therefore, is likely to be associated with an increase in dissolution rate through increased disruption of the particulate system allowing a particle greater movement and exposure to fresh dissolution medium, rather than any increase in relative-velocity value for a theoretical independent particle. Finally, in terms of particles held at the upper or lower cell boundary due to buoyancy forces, while the particle remains static, absolute fluid velocity, which changes with flow rate, is relevant. Once the particle is suspended in the fluid, relative velocity is the relevant parameter.Therefore, particle dissolution is also affected by flow rate when buoyancy forces dictate that a particle is static and its dissolution subject to absolute-velocity forces.

The above analysis (eq 2) is for the simplified case of spherical particles, whereas particle velocity also depends to an extent on particle shape. The concept of a particle shape factor (PSF) was introduced to adjust for the surface area of a nonspherical particle (13). An approximate PSF can be calculated by calculating the surface area of the particle relative to the surface area of a sphere of equivalent volume. As yet, however, there is no simple equation to predict the effect of particle shape on the effective diffusion boundary layer thickness.

\section{Imaging Techniques}

Overall particle motion effects such as cake formation, floating particles, or particles trapped at the upper filter can be observed by simple visualization. In terms of characterizing particle size and motion over the course of a dissolution test, a shadowgraph imaging method was recently introduced (8). Figure $2 \mathrm{~b}$ shows a typical shadowgraph image, in this case with the particle moving directly upward. The red arrow depicts both direction and velocity magnitude, and the particle diameter can also be measured (indicated here by the red circles). Out-of-focus particles are detected by the algorithm and omitted from the statistics.
With the shadowgraph imaging technique, particle direction, velocity, and diameter can be measured. From this, the change in particle size distribution over time can be observed. This observation is made within the sampling region only, and as demonstrated recently (8), the particles observed within the sampling region may represent a "window" of particle size distribution, with particles entering and leaving that window as they decrease in size.

Furthermore, the absolute lower particle diameter limit for particle detection with the optical arrangement (i.e., combination of a 105-mm macro lens with a 150-mm long extension tube) employed in the study by D'Arcy and Persoons (8) was $6 \mu \mathrm{m}$. Nevertheless, with this system a practical lower limit of approximately $30-\mu \mathrm{m}$ particle diameter is more realistic for dissolution imaging, to detect changes in particle diameter over time. Higher magnification lenses and higher resolution cameras are available, however, and this aspect should be explored along with method optimization. In summary, the shadowgraph imaging method could be further optimized to detect smaller particles and to look at a range of regions in the cell to detect overall particle velocities present and the overall change of particle size distribution with time. Knowledge of particle size and motion over the dissolution test can indicate whether the particles are mostly free to move with the fluid, thereby subject to a relative-velocity value that does not change with flow rate, or are somehow prohibited from moving freely (e.g., due to cake formation or particle density, resulting in the particle resting at the cell of the base during low-velocity periods of the pulse). In the latter situations where the particles are not free to move independently, the absolute-velocity value is relevant to the dissolution rate, and therefore dissolution of the exposed surface could be expected to vary with flow rate.

Shadowgraph imaging is a promising tool to investigate the hydrodynamic environment of a particulate dissolution system during dissolution testing in USP dissolution Apparatus 4, giving information on particle size and velocity. However, in a situation where there is cake formation, it is not only the particle motion and exposed surface area that are inhibited through contact with adjacent particles. The local concentration gradient is also affected, as the bulk concentration could not always be expected to be zero, despite the flow of fresh dissolution medium through the cell. During the zero and low-velocity period of the pulse, there is no, or limited, inflow of fresh dissolution medium. Furthermore, in considering a bed or cake of particles, not all areas of the surface are equally exposed to the fresh dissolution medium flowing through the cake, depending on the path of the medium. Therefore, local concentration gradients affect the dissolution rate. There have been some interesting developments in using UV imaging technology to analyze concentration gradients at a dissolving surface in a flow-through cell. 
Recently, it was reported (14) that quantitative analysis of concentration gradients is possible when investigating nicotine release from a nicotine patch using UV imaging. Although, to our knowledge, these methods have not been applied routinely to concentration gradients around particulate systems in USP Apparatus 4, the development of such techniques is promising for investigating the effects of local concentration gradients on dissolution rates. This is relevant also to nondisintegrating dissolving surfaces, for example to determine the effects of solution density on local hydrodynamics and its change with flow rate.

\section{CONCLUSION}

Investigation and simulation of hydrodynamic effects in the low-velocity, pulsing flow of USP dissolution Apparatus 4 can give some insight on how the hydrodynamics within the flow-through cell can affect the dissolution process. Relevant hydrodynamic features include the bulk pulsing flow field, which consists of periods of low to zero fluid velocity and periods of higher velocity depending on the flow rate. The velocity magnitude of this pulsing flow can be significant for distribution of a particulate system within the cell and consequent exposure of particles to fresh dissolution medium. Local hydrodynamic features, which may require simulation or imaging techniques to analyze, can result in fluid flow opposite to the bulk flow direction. This occurs due to effects of boundary layer separation and reversal at a dissolving surface and gravity induced natural convection caused by density gradients. Furthermore, significant variation in radial velocities and flow variability depending on tablet location and cell size were observed (4). Such local flow features can affect the local concentration gradient and thus the dissolution rate, in what may be an unpredictable manner, resulting in dissolution rates that do not increase with increasing flow rates under certain conditions. Although simulation tools such as CFD can be of assistance in predicting the hydrodynamic effects on dissolution, newer imaging applications such as MRI, shadowgraphy, and UV imaging show promise in their ability to determine the environment local to the dissolving surface. In the complex hydrodynamic environment of the low-velocity pulsing flow within USP Apparatus 4, interpretation of dissolution rate data requires consideration of hydrodynamics local to the dissolving surface in addition to overall flow rate.

\section{ACKNOWLEDGMENT}

This research was funded in part by the Irish Research Council for Science Engineering and Technology.

\section{REFERENCES}

1. Kakhi, M. Classification of the flow regimes in the flowthrough cell. Eur. J. Pharm. Sci. 2009, 37 (5), 531-544.

2. Kakhi, M. Mathematical modeling of the fluid dynamics in the flow-through cell. Int. J. Pharm. 2009, 376 (1-2), 22-40.

3. D'Arcy, D. M.; Liu, B.; Bradley, G.; Healy, A. M.; Corrigan, O.I. Hydrodynamic and species transfer simulations in the USP 4 dissolution apparatus: considerations for dissolution in a low velocity pulsing flow. Pharm. Res. 2010, 27 (2), 246-258.

4. Shiko, G.; Gladden, L. F.; Sederman, A. J.; Connolly, P.C.; Butler, J. M. MRI Studies of the Hydrodynamics in a USP 4 Dissolution Testing Cell.J.Pharm.Sci.2011, 100 (3), 976-991.

5. D'Arcy, D. M.; Corrigan, O. I.; Healy, A. M. Evaluation of hydrodynamics in the basket dissolution apparatus using computational fluid dynamics-dissolution rate implications. Eur. J. Pharm. Sci. 2006, 27 (2-3), 259-267.

6. The United States Pharmacopeia and National Formulary USP 34-NF 29; The United States Pharmacopeial Convention, Inc.: Rockville, MD, 2011.

7. European Pharmacopoeia, 7th ed.; European Directorate for the Quality of Medicines \& Healthcare, Council of Europe: Strasbourg, France, 2011.

8. D'Arcy, D. M.; Persoons, T. Mechanistic modelling and mechanistic monitoring: Simulation and shadowgraph imaging of particulate dissolution in the flow-through apparatus. J. Pharm. Sci. 2011, 100 (3), 1102-1115.

9. White, F. M. Viscous Fluid Flow, 2nd ed.; McGraw-Hill: NY, 1991.

10. Cammarn, S. R.; Sakr, A. Predicting dissolution via hydrodynamics: salicylic acid tablets in flow through cell dissolution. Int. J. Pharm. 2000, 201 (2), 199-209.

11. Stevens, L. E.; Missel, P. J. Impact of density gradients on flow-through dissolution in a cylindrical flow cell. Pharm. Dev. Technol. 2006, 11 (4), 529-534.

12. D'Arcy, D. M.; Liu, B.; Corrigan, O. I. Investigating the effect of solubility and density gradients on local hydrodynamics and drug dissolution in the USP 4 dissolution apparatus. Int. J. Pharm. 2011, 419 (1-2), 175-185.

13. Sugano, K. Introduction to computational oral absorption simulation. Expert Opin. Drug Metab. Toxicol. 2009, $5,(3), 259-293$.

14. Ostergaard, J.; Meng-Lund, E.; Larsen, S. W.; Larsen, C.; Petersson, K.; Lenke, J.; Jensen, H. Real-time UV imaging of nicotine release from transdermal patch. Pharm. Res. 2010, 27, 2614-2623. 